\title{
sciendo
}

Transport and Telecommunication, 2021, volume 22, no. 1, 15-28

Transport and Telecommunication Institute, Lomonosova 1, Riga, LV-1019, Latvia

DOI 10.2478/ttj-2021-0002

\section{GIS-BASED URBAN ROAD NETWORK ACCESSIBILITY MODELING USING MLR, ANN AND ANFIS METHODS}

\author{
K. Sai Sahitya ${ }^{1}$, CSRK Prasad ${ }^{2}$ \\ ${ }^{1}$ Transportation Division, Department of Civil Engineering, \\ National Institute of Technology, Warangal, Telangana, India \\ ksspvpcivil@gmail.com \\ ${ }^{2}$ Transportation Division, Department of Civil Engineering, \\ National Institute of Technology, Warangal, Telangana, India \\ csrkprasad@yahoo.com
}

\begin{abstract}
A sustainable transportation system is possible only through an efficient evaluation of transportation network performance. The efficiency of the transport network structure is analyzed in terms of its connectivity, accessibility, network development, and spatial pattern. This study primarily aims to propose a methodology for modeling the accessibility based on the structural parameters of the urban road network. Accessibility depends on the arrangement of the urban road network structure. The influence of the structural parameters on the accessibility is modeled using Multiple Linear Regression (MLR) analysis. The study attempts to introduce two methods of Artificial Intelligence (AI) namely Artificial Neural Networks (ANN) and Adaptive network-based neurofuzzy inference system (ANFIS) in modeling the urban road network accessibility. The study also focuses on comparing the results obtained from MLR, ANN and ANFIS modeling techniques in predicting the accessibility. The results of the study present that the structural parameters of the road network have a considerable impact on accessibility. ANFIS method has shown the best performance in modeling the road network accessibility with a MAPE value of $0.287 \%$. The present study adopted Geographical Information Systems (GIS) to quantify, extract and analyze different features of the urban transportation network structure. The combination of GIS, ANN, and ANFIS help in improved decision-making. The results of the study may be used by transportation planning authorities to implement better planning practices in order to improve accessibility.
\end{abstract}

Keywords: accessibility, GIS, road network, ANN, ANFIS

\section{Introduction}

An efficient transport network structure is responsible for a region's economic growth. The engineer's adherence towards a region can be understood by analyzing the efficiency of the transport network structure. A well-arranged and well-organized transportation network structure is very important for the development of a region. Good transportation network structure improves the accessibility thereby decreasing the travel time and travel cost. In an urban road network, the real difficulty lies in analyzing the effect of implemented policies on it. In the course of time, the changes that are made to the existing road network system influence the land use pattern and also travel demand in that particular region. The evaluation and analysis of basic structural measures like connectivity, accessibility, morphology and density of the transport network system are on focus in many studies. But the relationship between these parameters is not addressed in many of the studies. At this point, a research gap lies in urban road network planning. To fill this gap, to some extent the present study focuses on examining the relationship between structural parameters of urban road networks like connectivity, accessibility, spatial pattern and road network density of the existing road network in Hyderabad city, India.

Nowadays specific integrated system like GIS is being availed in urban road network planning to enhance the performance and quality of the transport structure. The present study implemented various tasks such as location finding, multiple ring buffer analysis, data extraction, data analysis using a GIS platform. The present work also explored the potentials of GIS in data capture, processing, and analysis in evaluating the structural parameters of urban road networks.

The present work attempted to develop models using MLR, ANN and ANFIS techniques to predict the urban road network accessibility based on connectivity, fractal dimension, and density. MLR analysis is used to establish a relationship between the road network structural parameters using accessibility in terms of MWAI as dependent and connectivity in terms of Aggregate Transportation Score (ATS), fractal dimension in terms of Mean Fractal Dimension (MFD) and road network development in terms of Mean 
Road Network Density (MRND) as independent variables. Artificial Neural Networks (ANN) technique is used in modelling the accessibility with MWAI as output variable and ATS, MFD and MRND as input variables in order to the urban road network accessibility. ANN is a method used to model the nonlinearity between the input and output variables. ANN is a method inspired by the biological neurons of human brains. It is a connected system of various layers of artificial neurons that transmits the signal from one neuron to the other. ANN can perform all the tasks a human brain can perform. ANN has a wide range of applications in the fields of engineering, regression analysis, pattern recognition, data processing, robotics, medicine etc. The adaptive network-based Neuro-Fuzzy inference system (ANFIS) method is also introduced in this study in order to predict the urban road network accessibility. ANFIS is a method that uses the process of neural networks and the rules of fuzzy-logic theory. The integration of these two methods yields remarkable results. ANFIS is also a method used to analyze the complex relationship between different input and output variables. The architecture of ANFIS comprises of five layers: first layer is fuzzification layer (input and membership functions), second is the rule layer (generating fuzzy rules), third is the normalizing layer (normalizing the values), the fourth one is the defuzzification layer (normalized values are returned to the output layer) and the fifth layer is the target or output layer.

This study aims to evaluate the existing road network structure in terms of ATS, MWAI, MFD, and MRND. This study also focuses on establishing the relationship of MWAI with ATS, MFD and MRND. The study attempts to introduce ANN and ANFIS modelling techniques to predict urban road network accessibility. The results of different modelling techniques (MLR, ANN and ANFIS) are analyzed and discussed.

\section{Literature Review}

This section describes the previous studies carried out on analyzing various road network structural parameters using GIS, accessibility modelling, and applications of ANN and ANFIS in the field of transportation.

The essential ingredients for any road network are connectivity, accessibility, hierarchy, and morphology. The ease of travel to desired destinations is facilitated in a well-connected street structure. Different modes of transport, different speeds, and volumes of vehicles are facilitated in a well-organized hierarchical road structure. The higher values of connectivity indicators represent a satisfactory interconnectedness of roads (Obafemi et al., 2011). A connectivity indicator is definitely needed to evaluate how well the street structure is organized and interconnected. This can be easily quantified by the ratio of vertices to the edges in a structure (Arora and Pandey, 2016). The connectivity indicators will act as a performance and efficiency base for many organizations that are helpful in the decision making process of transportation planning (Nijagunappa et al., 2007). There are three important indicators measuring connectivity like Alpha, Beta, and Gamma. With the help of these indicators, the transport structure performance based on the connectivity of roads can be explained easily (Kansky, 1963).Coverage measures describe the density aspect of the elements of a network, as intersections and links. Higher the value, the more the network is developed. The growth of the transport network is described based on the network length (Levinson, 2012) and network density (Bento et al., 2003). Network density and intersection density attribute network coverage in an area. Fractal geometry is an efficient approach to explain the complexity of the spatial objects. It is used to characterize irregularity and to quantify forms that are seemingly chaotic (Falconer, 1963, Hastings and Sugihara, 1993, DeCola and Lam, 1993, Manderbolt, 1982). The Fractal dimension of a "fractal pattern is a ratio that provides a statistical index of complexity comparing how details in a pattern change with the scale at which it is measured" (Falconer, 2003). A city is to be treated as distributive continuous space and the fractal analysis should be deployed on every piece of space on a GIS platform. A new method named Distributive Continuous Fractal analysis is introduced to evaluate the road networks (Sun et al., 2007).Fractal modeling is a mathematical tool applied to analyze network, functionality and built-up areas with a relational view and policy view for urban planning to generate a sustainable level of service of traffic, environment, and uniform road utility (Mohammadi et al., 2013). The fractal dimension of the transport network can be determined by a number of methods like the structured walk/divider method, equipaced polygon method, cell count method, mass-radius method, hybrid walk method, calliper method, pixel-dilation method, and box-counting method (Sreeleka et al., 2017). Accessibility is defined in many ways and by many researchers such as "the potential of opportunities for interaction" (Hansen, 1959), "the ease with which any land use activity can be reached from a location using a particular transportation system" (Dalvi and Martin, 1976), "the freedom of individuals to decide whether or not to participate in different activities" (Burns, 1979), and the benefits provided by a transportation or land use system". In the context of transport studies, accessibility indicates the ability of people to reach their 
destinations which they must visit in order to meet their needs, and desire to visit to fulfill their wants. All accessibility measures have two major components: the first is the attractiveness component and the second is the impedance function. The attractiveness component is usually measured as the number of opportunities at destinations. The impedance function decreases the probability of being attracted to such destinations based on distance or travel time. Accessibility often incorporates land-use patterns, network topology, and travel behavior. Accessibility measure is modeled as a utility-based measure (Avika and Lerman, 1977), topological measure (Mackiecvicz and Ratajczak, 1996), gravity measure (Hansen, 1959), cumulative opportunity measure (Ahmed et al., 2006), constrained based measure (Wu and Miller, 2002). There were some reviews on different accessibility measures with mathematical formulations and the reviews represented that, to determine the land use and transportation performance accessibility is the essential measure (Bhat et al., 2002). A facility-based planning methodology for rural roads has been proposed using spatial techniques. The accessibility for different facilities such as education facilities, medical facilities, economic activities and transport and communication facilities is quantified and analyzed in terms of Village Facility Index (VFI). A GIS-based rural road database was developed to improve the accessibility for different facilities from habitations and expand the infrastructure for current and future requirements (Modinpuroju et al., 2016). The interdependency between different urban road network structural parameters is modelled and analyzed using Arc GIS software. This study tried to illustrate that the road network structural parameters have some significant relationship with each one of them (Sahitya and Prasad, 2019). A city-level road network accessibility evaluation has been done based on the number of opportunities (or) urban services with respect to the distance using Arc GIS software. The level of accessibility has been categorized and the different locations of the study area were evaluated according to it (Sahitya and Prasad, 2020).

In the present day, the professionals of transportation are investigating the applications of Artificial Intelligence (AI) to solve some of the transportation problems in order to improve the performance of the transportation system. The transportation systems are very complex in nature with a lot of uncertainty and are difficult to analyze the conventional methods (TRB circular E-C113, 2007). The application of AI techniques had been extended to various sectors of transportation such as traffic flow operations, travel demand modelling and forecasting, transportation safety and security analysis, analysis of public transportation planning and operations and design and construction of transportation infrastructure (TRB circular E-C168, 2012). AI has various methods and applications in the field of transportation out of which ANN and ANFIS techniques are focussed on in this study. ANN was first introduced by (Culloch and Walter, 1943). ANN has an extensive usage in the field of transportation with a specific range of applications such as short term traffic flow prediction (Voort et al., 1996), time-delay neural network modelling (Abdulhai et al., 1999), estimating route travel times between individual localities in a urban traffic network (Fu and Rillet, 2000), vehicle delay modelling (Murat, 2006), planning of transportation network development in response to new developments (Król, 2016) and accident data analysis (Morris et al., 2017). ANFIS is the hybrid method of neural networks and fuzzy modelling. Fuzzy model (or) fuzzy identification was first studied methodically by (Takagi and Sugeno, 1985). ANFIS technique also has a wide range of its application in transportation environment in particular areas such as vehicle delay modelling (Murat, 2006), resource allocation, network optimum path analysis, sequential decision-making process etc., (TRB circular E-C113, 2007), short term traffic flow prediction (Bao-ping and Zeng-qiang, 2009), modelling and prediction of car-following behaviour in real traffic flow (Khodayari et al., 2010), evaluation of construction sustainability in highway design (Thipparat and Thaseepetch, 2012), development of trip generation model (Chauhan, 2013), road accident prediction (Hosseinpour et al., 2013), urban growth modelling (Mohammady, 2016), and predicting road network suitability mapping (Bugday, 2018). ANFIS also may be applied in the analysis of vehicle routing, traffic control at intersections with light signalling, vehicle steering and control, safety, modelling of fuel consumption, engine performance and exhaust emissions and traffic congestion (Stojcic, 2018). With the background of the aforementioned literature and understanding the feasibility of the application of different methods of AI in the field of transportation, this study attempted to introduce the concept of AI in urban road network accessibility modelling.

In recent years, there is a considerable concurrence of AI and GIS. GIS professionals started to study the non-parametric methods that may illustrate and model the data. "AI provides sophisticated techniques for GIS projects while GIS is a powerful technology with the vast data sets and a wide scope of applications for AI". The combination of AI and GIS is a useful tool for enhancing the quality of decision-making (Vozenilek, 2009). GIS experts can add another measure to their geographical abilities with the integration of AI (Holt and Benwell, 1999). The field of transportation has very less research with the combination of AI and GIS (Gopal, 2018). 


\section{Methodology}

The digitized and geo-referenced road network base map of Hyderabad city was obtained from HUDA (Hyderabad Urban Development Authority) office. The digitized road network map was used for quantifying the structural parameters of the urban road network. In the first stage, thirty-six locations of Hyderabad city, one location for every 200 sq. km of road network area were selected covering the entire city in all directions. The buffer zones were developed form the centre of each location with a radius of 1$5 \mathrm{~km}$ using multiple ring buffer analysis tools in Arc GIS 10.1 software. Then the connectivity, accessibility, road network density and fractal dimension values were computed for all locations at different radii. The study intended to predict the accessibility of road network with respect to the other structural parameters. In the second stage, the accessibility was modelled using MLR, ANN and ANFIS modelling techniques. In the later stage, the results obtained from different modelling techniques were compared and analyzed. The methodology followed in the study is presented in Figure. 1. The various methods used in this study are described in the following sections.

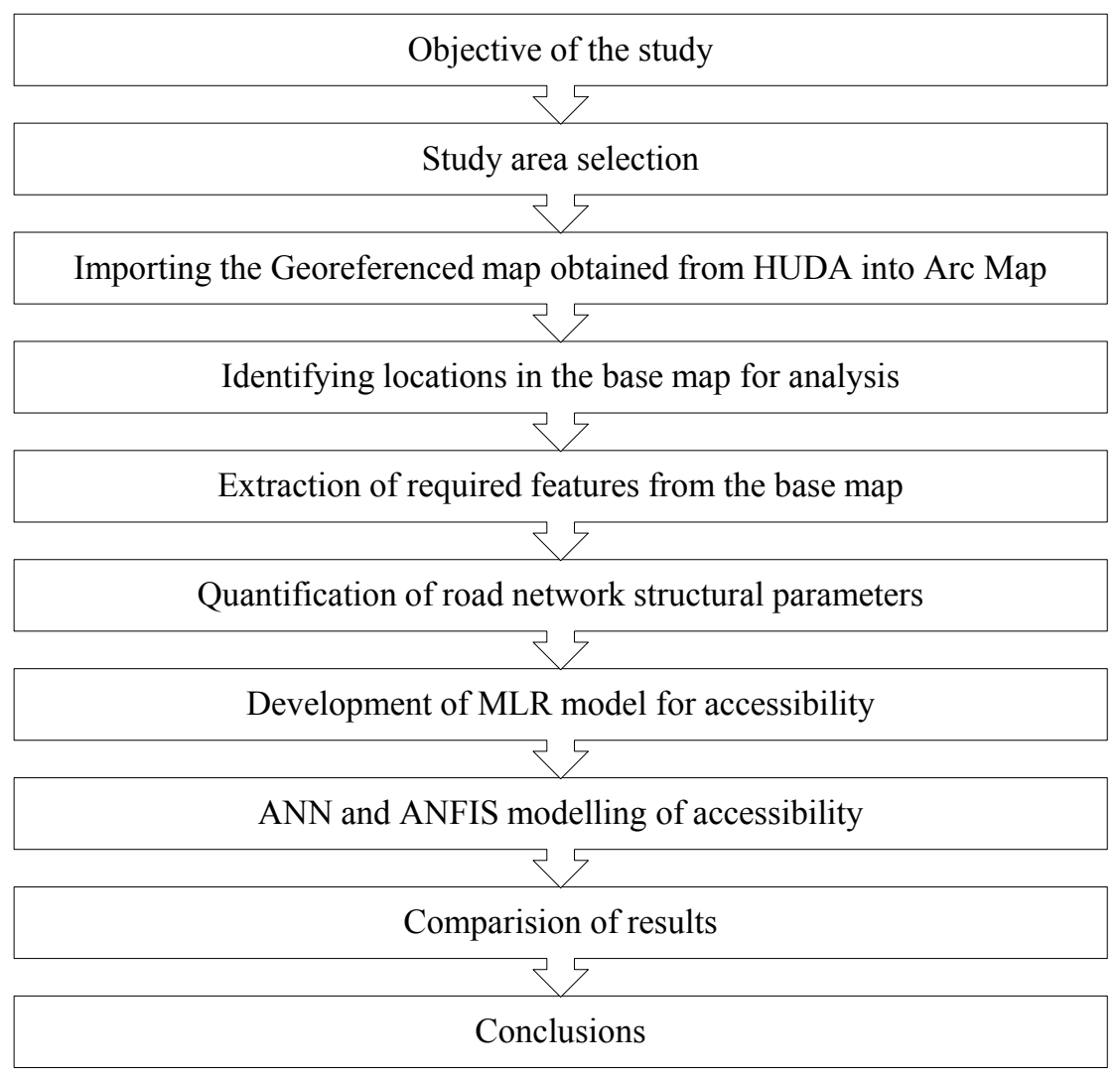

Figure 1. Flow chart showing the methodology of the study

\subsection{Study Area}

Hyderabad city, Capital of Telangana state, India is chosen as a study area for the present study. The total road network length 5400 kilometres and a road network area of $7200 \mathrm{sq} . \mathrm{km}$. The location of the study area is shown in Figure 2. Thirty-six locations, one for each $200 \mathrm{sq} . \mathrm{km}$ of road network area are selected covering the entire city in all directions. The locations selected for this study are Abids (AB), Kukatpally (KP), Mehadipatnam (MP), Dilshuknagar (DN), Secundrabad (SC), Panjagutta (PG), Jeedimetla (JM), Sikenderguda (SG), Nadergul (NG), Kothapet (KT), Ameerpet (AT), Banjara Hills (BH), Gandhi Nagar (GN), LB Nagar (LN), Malkajgiri (MJ), Hitech city (HC), Bhuvanagiri (BG), Ghatkesar (GS), Bhanur (BR), Moinabad (MB), Rama Chandra Puram (RCP), Shivampet (SP), Sangareddy (SR), Wargal (WL), Domara Pocham Pally (DPP), Sitharampuram (SM), Kothur (KR), Golconda Khurd (GK), Thummaloor (TR) Ibrahimpatnam (IBM), Ramalingapally (RL), Jagathpally (JP), Chevella (CV), Shahabad (SH), Kallakal (KL) and Yellammaguda (YL). The locations chosen for the 
analysis are shown in Figure 3. The legend of Figure 3 represents the colours of multiple rings created for each kilometre radius $(1 \mathrm{~km}, 2 \mathrm{~km}, 3 \mathrm{~km}, 4 \mathrm{~km}$, and $5 \mathrm{~km})$.

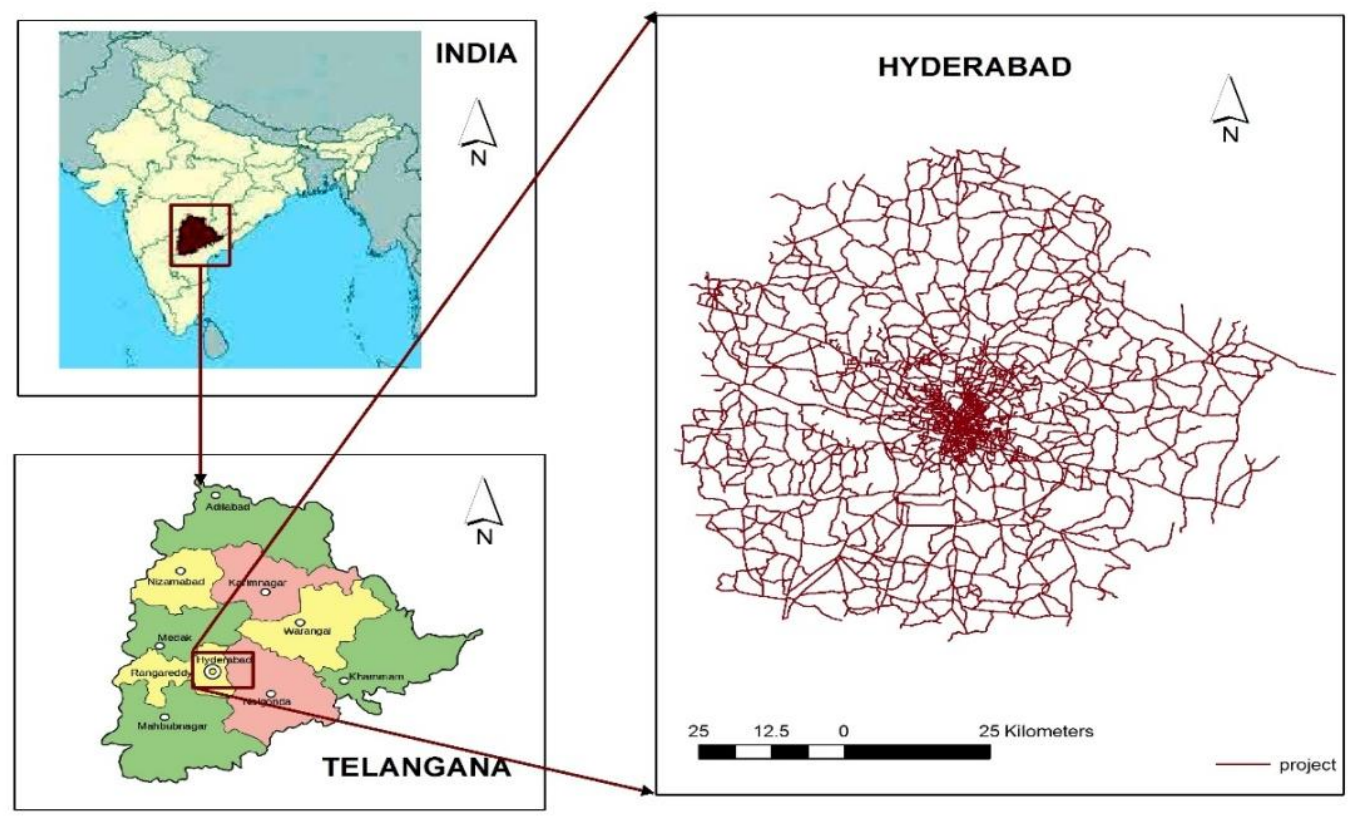

Figure 2. Location of the study area

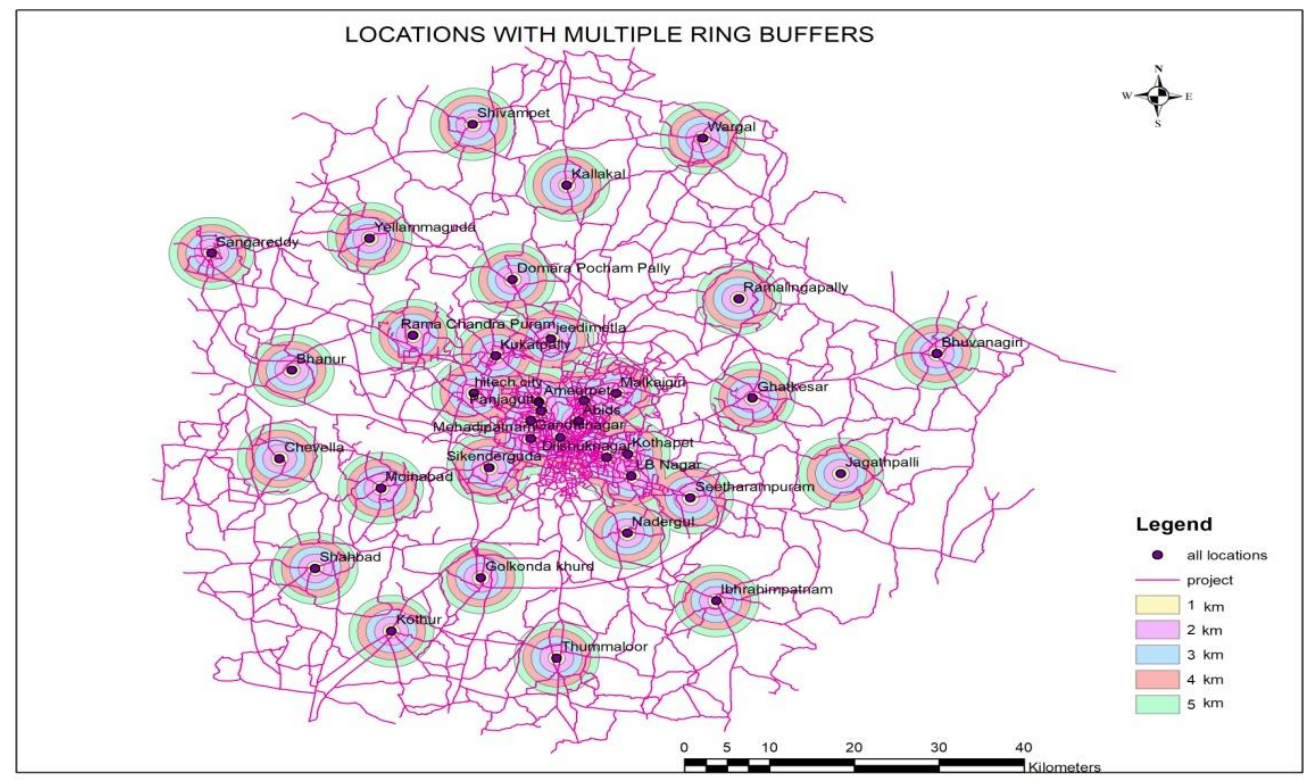

Figure 3. Map showing the locations chosen for the analysis

\subsection{Road network structural analysis}

Road network structure can be analyzed using four major parameters namely connectivity, accessibility, spatial pattern, and network development. All the features required for the analysis such as number of nodes, number of links, road network length, road network area, built-up area, number of opportunities (or) urban services and distances are extracted using Arc GIS 10.1 software. The methods adopted to quantify each of these parameters are described here. 


\subsubsection{Connectivity}

The study availed graph theory measures like alpha index $(\alpha)$, beta index $(\beta)$, gamma index $(\gamma)$, Cyclomatic number $(\mu)$, and ATS to analyze the road network connectivity. These measures are quantified for all locations at each radius. The greater the value of these indices, the higher the connectivity of the road network. ATS is the sum of the alpha index, beta index, gamma index, and Cyclomatic number. Therefore, ATS can be able to interpret the overall connectivity of a region or location. The connectivity measures are computed using Equation (1) to Equation (5), where equations are:

$\alpha=(e-v+1) /(2 v-5)$,

$\beta=e / v$,

$\gamma=e / 3(v-2)$

$\mu=e-v+1$,

ATS $=\alpha+\beta+\gamma+\mu$,

where $e=$ number of edges/ links and $v=$ number of nodes/ vertices.

\subsubsection{Accessibility}

In this study, the accessibility of the road network in the study area is measured in terms of the Mean Weighted Accessibility Index (MWAI). Accessibility index is quantified with respect to distance and the number of opportunities. The number of various opportunities or urban services like schools, colleges, ATM's, banks, hospitals, police stations, petrol bunks, bus-stops, parks, and theatres are computed at each radius (0-1, 1-2, 2-3, 3-4, 4-5kilometres) for different locations. The weights are given to each type of opportunity and the accessibility index for each buffer radius is computed. Then the MWAI is quantified for each location. MWAI of a location is the mean of the weighted accessibility indices obtained at different buffer radius. MWAI of a location is the mean of the weighted accessibility indices obtained at different buffer radius. The accessibility index $\left(A_{i}\right)$ is computed using Equation (6) where accessibility index is:

$A_{i}=\Sigma\left(W_{i} \times N_{i r}\right)$,

where $W_{i}=$ Weights assigned to $i^{\text {th }}$ opportunity (or) urban service and $N_{i r}=$ Number of $i^{\text {th }}$ opportunity at $r^{\text {th }}$ radius.

\subsubsection{Spatial Pattern (or) Fractal dimension}

The spatial pattern of the existing transport network is quantified using fractal geometry. The mass-radius fractal method is used in this study to analyze the fractal dimension of the road network. The fractal dimension (D) is quantified with respect to the number of nodes, road network length and built-up area for all locations in each buffer radius. Then the spatial pattern of a location is quantified in terms of MFD. MFD of a location is the mean of all the fractal dimension values obtained in different radius. The fractal dimension $\left(D_{i}\right)$ is computed using Equation (7) and the fractal dimension is:

$D_{i}=\log \left[x\left(R_{i}\right) / x\left(R_{i-1}\right)\right] / \log \left[R_{i} / R_{i-1}\right]$,

where $R_{i}$ is the radius at scale $i, \mathrm{X}$ is the number of nodes (n) (or) road network length (l) (or) built-up area (a) at scale $i, R_{i-1}$ is the radius of scale $i-1, x\left(R_{i-1}\right)$ is the number of nodes (or) road network length (or) built-up area at the scale $i-1$ of and $D_{i}$ is the fractal dimension of nodes (or) road network length (or) built-up area at scale $i$.

\subsubsection{Road network development (or) network density}

Road network development is quantified in terms of road network density (RND). The length and area of the road network are computed for all the locations in each buffer radius. Higher the density, the higher the network development. In this study, the area of the zone is considered as the area of the buffer for calculating the road network density. The road network development for a location is computed in terms of MRND. MRND of a location is the mean of the network density values obtained in the different radius of each location. The road network density is calculated using Equation (8) and road network density is: 
$R N D=R N L / A$,

where $R N D=$ Road Network Density, $R N L=$ Road network length $(\mathrm{km})$ and $A=$ Area of the zone (sq.km).

\subsection{Modelling the road network accessibility}

This phase of the study deals with the prediction of road network accessibility with respect to the other three structural parameters. Various modelling techniques such as MLR, ANN and ANFIS are involved in order to predict the accessibility. MLR method is used to establish a relationship between road network structural parameters with accessibility as the dependent variable and the other three as independent variables. ANN and ANFIS methods are used to identify the non-linearity between the input and output variables. The output variable considered here is accessibility and the remaining three variables are considered as input variables. The results of these methods are compared with respect to different statistical measures.

\section{Results and Discussions}

This section presents the results obtained in various stages of the study like road network structural analysis and modelling the accessibility.

\subsection{Road network structural analysis}

The connectivity in terms of ATS, accessibility in terms of MWAI, spatial pattern in terms of MFD, road network development in terms of MRND was computed for all locations. The descriptive statistics of ATS, MWAI, MFD and MRND are presented in Table 1.

Table 1. Descriptive statistics of road network structural analysis

\begin{tabular}{|c|c|c|c|c|c|}
\hline Location & Abbreviation & ATS & MWAI & MFD & MRND \\
\hline Mehadipatnam & MP & 73.65 & 20.00 & 1.00 & 3.89 \\
\hline Dilshuknagar & $\mathrm{DN}$ & 76.18 & 22.68 & 1.20 & 4.38 \\
\hline Secundrabad & $\mathrm{SC}$ & 68.67 & 16.78 & 1.40 & 4.54 \\
\hline Jeedimetla & $\mathrm{JM}$ & 20.32 & 10.43 & 1.13 & 1.22 \\
\hline Sikenderguda & $\mathrm{SG}$ & 20.01 & 6.10 & 1.15 & 1.10 \\
\hline Ameerpet & $\mathrm{AT}$ & 56.76 & 19.40 & 1.30 & 3.42 \\
\hline Banjara Hills & $\mathrm{BH}$ & 66.16 & 27.52 & 1.13 & 3.73 \\
\hline Gandhi nagar & GN & 104.18 & 25.18 & 1.48 & 5.13 \\
\hline LB Nagar & $\mathrm{LN}$ & 35.87 & 30.64 & 0.90 & 2.16 \\
\hline Hitech city & $\mathrm{HC}$ & 25.79 & 17.28 & 1.38 & 3.16 \\
\hline Ghatkesar & GS & 16.11 & 5.10 & 0.40 & 1.24 \\
\hline Ramalingapally & $\mathrm{RL}$ & 17.25 & 4.28 & 0.68 & 2.24 \\
\hline Jagatthpalli & JP & 5.25 & 5.12 & 0.89 & 4.10 \\
\hline Bhanur & $\mathrm{BR}$ & 4.26 & 1.50 & 1.08 & 0.74 \\
\hline Moinabad & $\mathrm{MB}$ & 6.40 & 3.20 & 0.70 & 1.37 \\
\hline Rama Chandra Puram & $\mathrm{RCP}$ & 15.71 & 10.18 & 1.50 & 0.94 \\
\hline Shivampet & SP & 3.63 & 0.29 & 0.45 & 0.79 \\
\hline Sangareddy & SR & 5.80 & 6.24 & 0.68 & 4.89 \\
\hline Shahabad & $\mathrm{SH}$ & 6.99 & 1.98 & 1.22 & 3.40 \\
\hline Kallakal & $\mathrm{KL}$ & 4.63 & 1.29 & 1.15 & 1.79 \\
\hline Sitharampuram & $\mathrm{SM}$ & 16.98 & 2.08 & 0.95 & 1.01 \\
\hline Kothur & $\mathrm{KR}$ & 5.99 & 0.98 & 0.75 & 2.40 \\
\hline Golconda Khurd & GK & 6.21 & 0.22 & 2.48 & 1.17 \\
\hline Ibrahimpatnam & IBM & 6.93 & 4.10 & 0.35 & 1.18 \\
\hline Abids & $\mathrm{AB}$ & 114.02 & 19.82 & 1.00 & 5.50 \\
\hline Kukatpally & $\mathrm{KP}$ & 25.12 & 16.66 & 1.25 & 2.24 \\
\hline Panjagutta & $\mathrm{PG}$ & 70.94 & 17.56 & 1.45 & 3.71 \\
\hline
\end{tabular}




\begin{tabular}{|l|l|l|l|l|l|}
\hline Location & Abbreviation & ATS & MWAI & MFD & MRND \\
\hline Nadergul & NG & 7.51 & 2.02 & 2.30 & 0.70 \\
\hline Kothapet & KT & 48.00 & 13.72 & 1.15 & 3.16 \\
\hline Malkajgiri & MJ & 48.94 & 25.06 & 1.25 & 2.69 \\
\hline Bhuvanagiri & BG & 4.43 & 4.20 & 0.65 & 4.09 \\
\hline Chevella & CV & 7.40 & 4.20 & 1.70 & 2.37 \\
\hline Wargal & WL & 13.68 & 0.62 & 1.83 & 2.76 \\
\hline Yellammaguda & YL & 6.80 & 7.24 & 1.08 & 5.89 \\
\hline Domara Pocham Pally & DPP & 16.33 & 1.20 & 1.50 & 0.88 \\
\hline Thummaloor & TR & 5.94 & 0.42 & 1.18 & 1.09 \\
\hline
\end{tabular}

\subsection{Modelling the road network accessibility}

\subsubsection{Multiple Linear Regression (MLR) modelling}

In this study, correlation and Multiple Linear Regression Analysis (MLR) were performed to examine the relationship between different structural parameters of the urban road network. Here, MWAI is considered as a dependent variable where ATS, MFD, and MRND are considered as explanatory variables. The change in accessibility with respect to the changes in connectivity, fractal dimension, and road network density is observed and modelled. The values of different structural parameters quantified for twenty-four locations are used in model development and the remaining twelve locations values are used for model validation. The model developed is validated for its adequacy.

\section{Correlation analysis}

Pearson's correlation analysis is performed to describe the relationship of MWAI with ATS, MFD, and MRND. The result of the correlation matrix is presented in Table 2.

Table 2. Correlation matrix

\begin{tabular}{|l|l|l|l|l|}
\hline & MWAI & ATS & MFD & MRND \\
\hline MWAI & 1 & & & \\
\hline ATS & 0.80 & 1 & & \\
\hline MFD & 0.90 & 0.64 & 1 & \\
\hline MRND & 0.91 & 0.52 & 0.51 & 1 \\
\hline
\end{tabular}

\section{Model development - Regression analysis}

Out of 36 locations, 24 (MP, DN, SC, JM, SG, AT, BH, NG, LN, HC, GS, RL, JP, BR, MB, RCP, $\mathrm{SP}, \mathrm{SR}, \mathrm{SH}, \mathrm{KL}, \mathrm{SM}, \mathrm{KR}, \mathrm{GK}, \mathrm{IBM}$ ) are chosen for model development. The model developed to estimate or predict the accessibility (MWAI) is presented in Equation (9) and the equation is:

$Y=2.82+0.274 \times A T S+0.75 \times M F D+0.164 \times M R N D$,

where $Y=$ Mean Weighted Accessibility Index (MWAI), ATS = Connectivity in terms of Aggregate Transportation Score, $M F D=$ Mean Fractal Dimension and $M R N D=$ Mean Road Network Density $(\mathrm{km} / \mathrm{sq} \cdot \mathrm{km})$.

The regression outputs presented in Table 3 indicates that all the variables used in developing the model can be included in the model because each variable has obtained a 'P-value' less than 0.05 and a't-stat' value greater than 1.96. This illustrates that the explanatory variables are significantly influencing the dependent variable. The intercept value of Table 3 represents the accessibility value when the remaining variables are zero. It is the constant vale obtained in MLR analysis.

Table 3. Regression analysis outputs

\begin{tabular}{|l|l|l|l|}
\hline Variable & Coefficient & t-stat & P-Value \\
\hline Intercept & 2.82 & 2.96 & 0.015 \\
\hline ATS & 0.27 & 7.90 & 0.0001 \\
\hline MFD & 0.75 & 2.98 & 0.002 \\
\hline MRND & 0.16 & 4.35 & 0.004 \\
\hline
\end{tabular}




\section{Model validation}

The model developed to predict the accessibility of different locations is validated for its adequacy. The values of MWAI, ATS, MFD, and MRND obtained for twelve locations (AB, KP, PG, $\mathrm{NG}, \mathrm{KT}, \mathrm{MJ}, \mathrm{BG}, \mathrm{CV}, \mathrm{WL}, \mathrm{YL}$, DPP, and TR) are used for model validation. The model validation presented that there is no significant difference between the measured and modelled MWAI values. The model validation is successful as it showed an RMSE value of 0.11 (which is less than 20\%) and a Pvalue of 0.09 (which is greater than 0.05 ). The relation between measured and modelled values is presented in Figure 4(a). The trend of measured and MLR modelled MWAI values for all locations are presented in Figure 4(b).
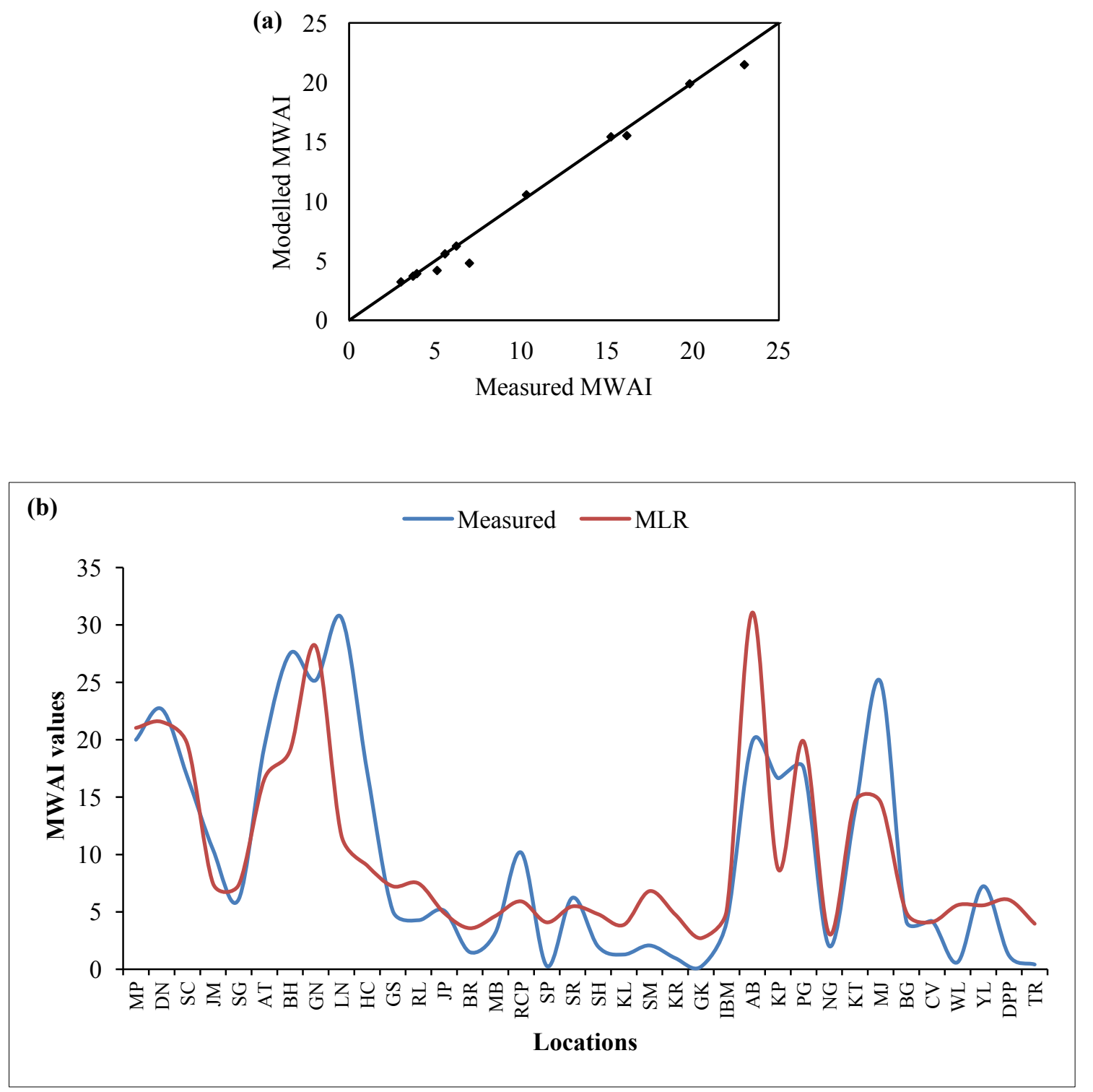

Figure 4. Measured v/s MLR modeled MWAI values (a) for locations selected for model validation and, (b) for all locations

\subsubsection{Artificial Neural Network (ANN) modelling}

The present study adopted the ANN approach to predict road network accessibility. Multi-layered feed-forward ANN is used in the present study for analysis. The study availed MATLAB for ANN modelling. ANN comprises three layers namely the input layer, hidden layer for calculating input weights and output layers. In this study, MWAI is given as output layer and ATS, MFD and MRND are given as input layers. ANN is developed in three steps. The first step is network training, the second step is the 
selection of optimal network structure and the third step is testing. ANN utilizes $70 \%$ of the data for training, $15 \%$ for testing and $15 \%$ for validation. The number of hidden layers and neurons are decided based on the trial and method iterations. The network structure with less error between the measured and ANN modelled values is chosen to be the optimal structure. In this study, the network structure for predicting the accessibility is optimized at 2 hidden layers and 10 neurons. The output of ANN modelling is presented in Figure 5(a). The trend of measured and ANN modelled MWAI values for all locations are presented in Figure 5(b).

(a)

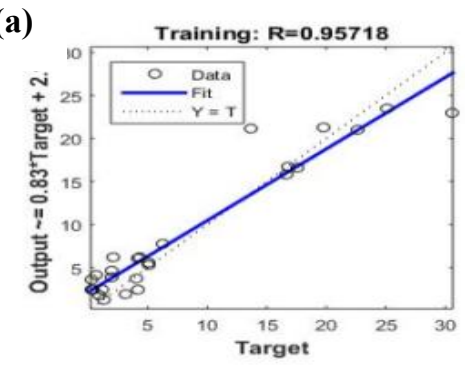

(c)

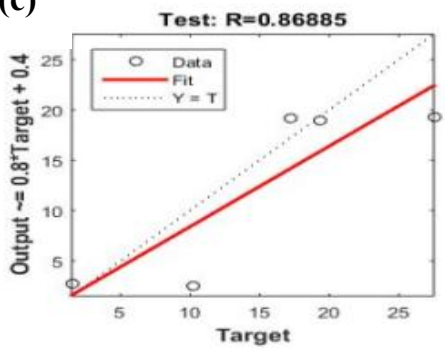

(b) Validation: $\mathbf{R}=0.92019$

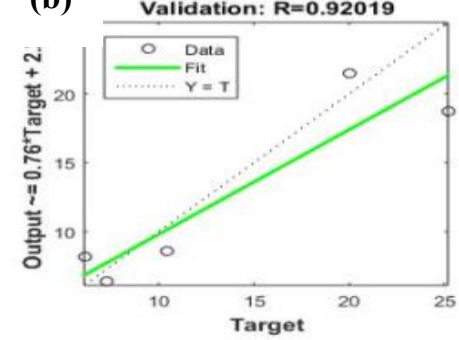

(d)

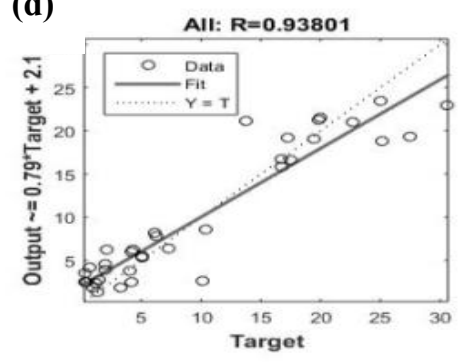

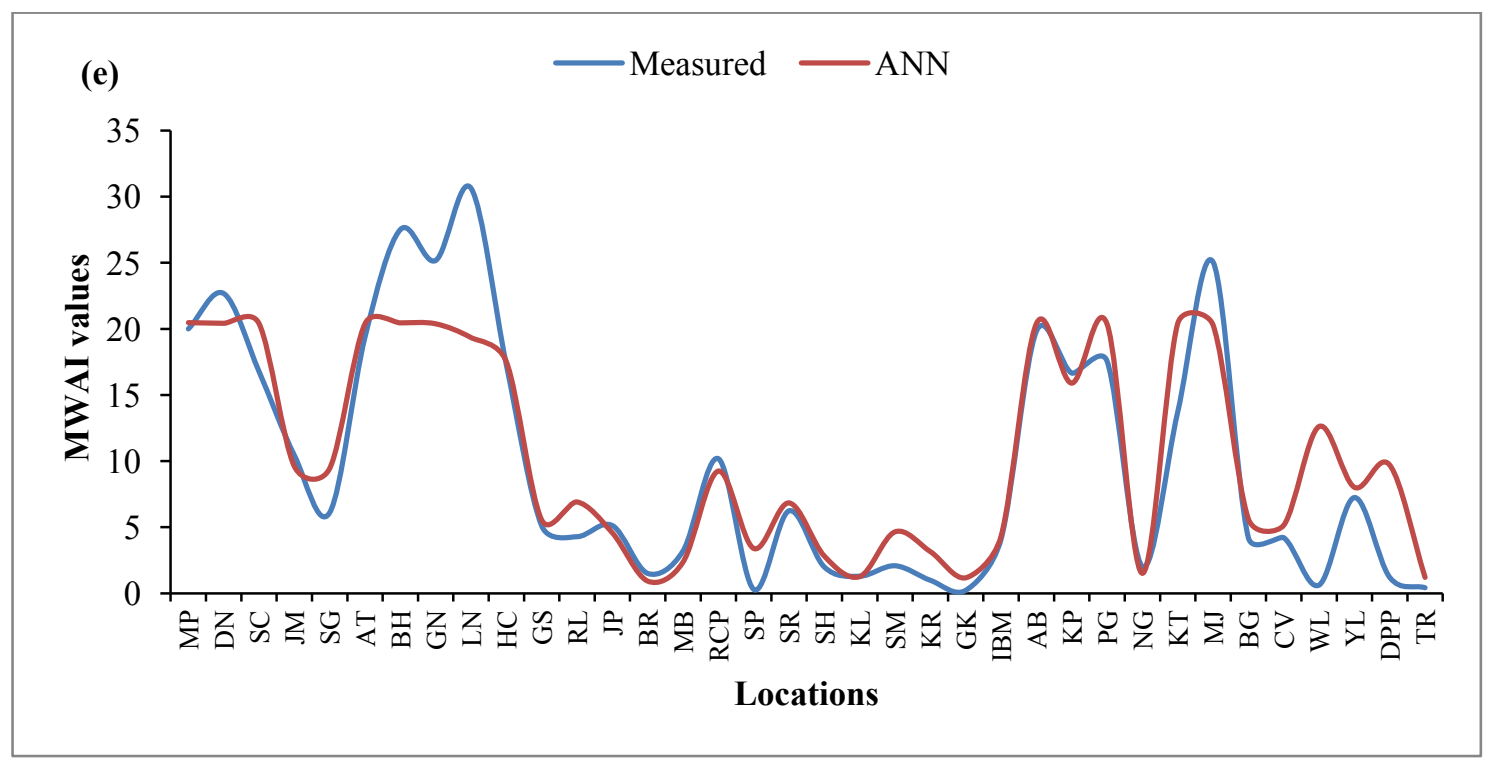

Figure 5. Output of ANN modeling (a) Training, (b) Validation, (c) Testing, (d) Combined, and (e) Measured v/s ANN modeled MWAI values

\subsubsection{Adaptive network-based Neuro-Fuzzy inference system (ANFIS) modelling}

This study also attempted to introduce the ANFIS approach in predicting the road network accessibility. ANFIS modelling is also performed using MATLAB Neuro-Fuzzy designer toolbox. ANFIS comprises three principal components: membership functions, fuzzy rules and output. ANFIS trains the given input data according to the fuzzy rules provided based on the membership function chosen. In this study, the ANFIS structure is provided with one output layer (MWAI) and three input 
layers (ATS, MFD, and MRND). This study used triangular membership function ('trimf') to train the data because it has shown better performance with a minimum percentage of error than the other membership functions like trapezoidal, Gaussian and bell. All possible if-then fuzzy rules are considered as input to provide the inference of the output. The prediction of MWAI using ANFIS modelling technique is shown in Figure 6.

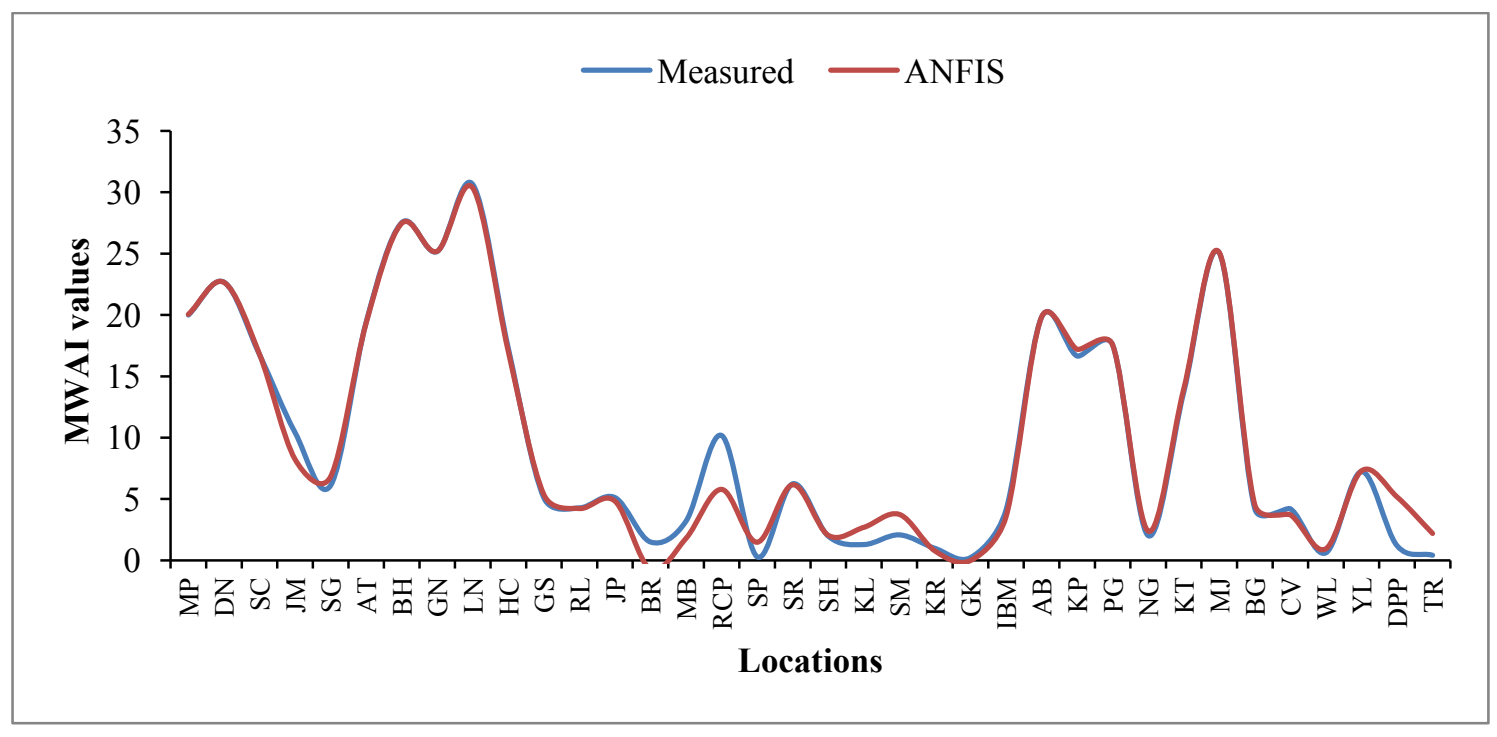

Figure 6. Measured v/s ANFIS modeled MWAI values

\subsubsection{Comparison of MLR, ANN, and ANFIS modelling techniques}

In this section, a comparison has been made between MLR, ANN and ANFIS modelling methods in predicting the road network accessibility. The measured MWAI and MLR, ANN and ANFIS modelled MWAI values are plotted in a graph and is presented in Figure 7. Fig.7 clearly depicts that the trend of measured MWAI values is very closer to the ANFIS modelled MWAI values. This indicates that the ANFIS modelling technique showed satisfactory performance when compared to the other two methods.

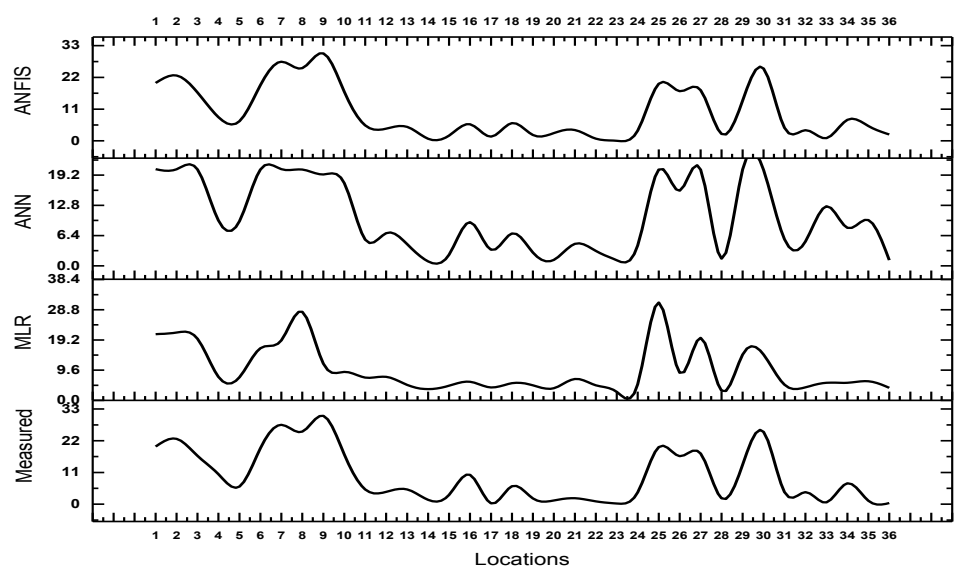

Figure 7. Comparison of MLR, ANN and ANFIS modeled MWAI values

In the next phase, the predicted MWAI values are checked for their accuracy using some statistical estimates. The error between the measured and modelled values is also quantified to examine the performance of different models. The statistical estimates such as $\mathrm{R}^{2}$ value, P-value, Root Mean Square Error (RMSE) and Mean Absolute Percentage Error (MAPE) are used for measuring accuracy and determining the error. The model with high $\mathrm{R}^{2}$ and P-values and low RMSE and MAPE values give the bestpredicted values. The statistical estimates of MLR, ANN and ANFIS techniques are shown in Table 4. 
Table 4. Statistical estimates for accuracy and error measurements

\begin{tabular}{|l|l|l|l|l|}
\hline Model & $\mathrm{R}^{2}$-value & P-value & RMSE & MAPE (\%) \\
\hline MLR & 0.72 & 0.09 & 0.112 & 1.526 \\
\hline ANN & 0.91 & 0.12 & 0.08 & 1.323 \\
\hline ANFIS & 0.96 & 1.01 & 0.007 & 0.287 \\
\hline
\end{tabular}

Table 4 clearly indicates that ANFIS has predicted the MWAI values to its best, because of its low RMSE and MAPE values and high $\mathrm{R}^{2}$ and P-values. This result shows that the ANFIS model is more efficient rather than the other models in predicting the road network accessibility.

\section{Conclusions}

The results of the study serve a variety of purposes, including quantification of urban road network connectivity, accessibility, fractal dimension, and density; determine a relation between urban road network structural parameters and to develop MLR, ANN and ANFIS models to predict the road network accessibility. The parameters like connectivity, accessibility, spatial pattern, and road network density are analyzed using Arc GIS 10.1 software. MLR modelling method is used to establish a relationship between different road network structural parameters. The regression equation developed in this study can be used to predict the road network accessibility at any location in Hyderabad city. Though the regression outputs of the MLR equation are in the standard limits, the results are not satisfactory. Therefore, this paper defined new methods in accessibility prediction modelling based on ANN and ANFIS. When these two methods are compared, the ANFIS model has shown acceptable performance. The statistical estimates obtained in the study proved the effectiveness of the ANFIS model in predicting the road network accessibility. This study helps the urban transportation planning authorities or people of the decisionmaking system to analyze the situations at different locations of the study area in order to optimize the road network in the city. The policy-makers can now decide to bring out policies that can create a balance between the road network's connectivity, accessibility, spatial pattern, and development. The study may be further extended to other urban cities including various other parameters like land-use, population density, etc. The integration of knowledge-based expert systems (GIS) and Artificial Intelligence (ANN and ANFIS) improves the potential in the decision making process of the transportation planners. The research trend of integrating GIS and AI has to be continued in road network structural modelling by many researchers (or) urban transportation planners in order to develop an efficient transportation system.

\section{Acknowledgments}

The authors are grateful to the HUDA authorities for providing the necessary data. The authors would also like to thank the anonymous editors and referees of the manuscript for their support in the improvement of the manuscript.

\section{References}

1. Abdulhai, B., Porwal, H., and Recker, W. (1999) Short-Term Freeway Traffic Flow Prediction Using Genetically Optimized Time-Delay-Based Neural Networks. Presented at 78th Annual Meeting of the Transportation Research Board, Washington, D.C. Report for MOU 360, ISSN 1055-1417.

2. Ahmed, Geneidy M.E.I., and David, M.L. (2006) Access to destinations: Development of accessibility measures. In a research report published by Minnesota Department of Transportation, Minnesota.

3. Arora, A., and Pandey, M.K. (2011) Transportation network model and network analysis of road networks. 12th Esri India User Conference 2011.

4. Avika, B., and Lerman. (1977) Disaggregate travel and mobility choice models and measures of accessibility. Behavioural Travel Modelling, eds. Hensherd and Stopher, P., London: Croom Helm, pp.654-679.

5. Bao-ping, C., and Zeng-qiang, M.A. (2009) Short-term Traffic Flow Prediction Based on ANFIS. In: International Conference on Communication Software and Networks, DOI 10.1109/ICCSN.2009.140.

6. Bento, A.M., Cropper, M.L., Mobarak, A.M., and Vinha, K. (2003) The impact of urban spatial structure on travel demand in the United States. World Bank Policy, 2003, Research Paper No. 3007. 
7. Bhat, C., Handy, S., Kockelamn, K., Mahmassani, H., Chen, Q., and Weston, L. (2002) Urban Accessibility index: Literature Review. Research Report TX 01/7-4938-01, Texas Department of Transportation, TX.

8. Bugday, E. (2018) Application of artificial neural network system based on ANFIS using GIS for predicting forest road network suitability mapping. Fresenius Environmental Bulletin, 27(3), 1656-1668.

9. Burns, L.D. (1979) Transportation, Temporal and Spatial Components of Accessibility. Lexington, MA: Lexington Books.

10. Chauhan, B. (2013) ANFIS based Trip Generation model for Meerut. International Journal of Computer Science and Mobile Computing, 2(12), 153-159.

11. Dalvi, M.Q., and Martin, K.M. (1976) The measurement of accessibility: Some preliminary results, Transportation, 5, 17-42.

12. De Cola, L., and Lam, N. (1993) Introduction to fractals in geography. Fractals in Geography (Prentice-Hall, Englewood Cliffs, NJ), 3-22.

13. Falconer, KJ. (1986) The geometry of fractal sets. Cambridge university press.

14. Falconer, KJ. (2003) Fractal Geometry - Mathematical Foundations and Applications, 2nd ed. Chichester: John Wiley and Sons, 338 p, ISBN 978-0-470-84862-3.

15. Fu, L., and Rilett, L.R. (2000) Estimation of Time-Dependent, Stochastic Route Travel Times Using Artificial Neural Networks. Transportation Planning and Technology, 24(1), 25-36.

16. Gopal, S. (2018) Artificial neural networks in geospatial analysis. The International Encyclopaedia of Geography, DOI: 10.1002/9781118786352.wbieg0322.

17. Hansen, W. (1959) How accessibility shapes land use. Journal of the American Institute of the Planners, 25, 73-76.

18. Hastings, H.M., and Sugihara, G. (1993) Fractals. A user's guide for the natural sciences. Oxford Science Publications, Oxford, New York: Oxford University Press, 1993, 1

19. Holt, A., and Benwell, G.L. (1999) Applying case-based reasoning techniques in GIS. International Journal of Geographical Information Science, 13(1), 9-25.

20. Hosseinpour, M., Yahaya, A.S., Ghadiri, S.M., and Prasetijo, J. (2013) Application of Adaptive Neuro-Fuzzy Inference System for Road Accident Prediction. KSCE Journal of Civil Engineering (2013) 17(7):1761-1772.

21. Kansky, K. (1963) Structure of Transportation Networks: Relationships between Network Geometry and Regional Characteristics. Ph. D. thesis, University of Chicago, Research Paper No. 84.

22. Khodayari, A., Ghaffari, A., Kazemi, R., and Manavizadeh, N. (2010) ANFIS based modelling and prediction car following behavior in real traffic flow based on instantaneous reaction delay. 13th International IEEE Annual Conference on Intelligent Transportation Systems Madeira Island, Portugal, September 19-22, 2010.

23. KrólA. (2016) The application of the artificial intelligence methods for planning of the development of the transportation network. Transportation Research Procedia, 14, $4532-4541$.

24. Levinson, D. (2012) Network Structure and City Size, Plos One, 7(1), DOI:10.1371/journal.pone.0029721.

25. Mackiewicz, A., and Ratajczak, W. (1996) Towards a new definition of topological accessibility. Transportation Research, Part B, 30(1), 47-79.

26. Mandelbrot, B.B. (1982) The fractal geometry of nature. 1982. In. WH Freeman \& Company.

27. McCulloch., Warren; Walter Pitts. (1943) A logical calculus of ideas immanent in nervous activity. Bulletin of Mathematical Biophysics, 5(4), 115-133. DOI: 10.1007/BF02478259.

28. Modinpuroju, A., Prasad, C.S.R.K., and Chandra, M. (2016) Facility-based planning methodology for rural roads using spatial analysis techniques. Innovative Infrastructure Solutions, 1(1), 1-8, DOI 10.1007/s41062-016-0041-8.

29. Mohammadi, A., Rao, K.M.L., and Baseer, M.A.K. (2013) Fractal view policy development on road infrastructure in urban areas. International Journal of Earth sciences and Engineering, 6(4), (01).

30. Mohammady, S. (2016) Optimization of adaptive neuro-fuzzy inference system based urban growth model. City, Territory and Architecture, 3, Article 10, pp. 1-15, DOI 10.1186/s40410-016-0039-8.

31. Morris, D., Antoniades, A., and Took, C.C. (2017) On making sense of neural networks in road analysis. International Joint Conference on Neural Networks (IJCNN), 4416-4421, DOI: 10.1109/IJCNN.2017.7966415.

32. Murat, Y.S. (2006) Comparison of fuzzy logic and artificial neural networks approaches in vehicle delay modelling. Transportation Research Part C 14, 316-334. DOI:10.1016/j.trc.2006.08.003 
33. Nijagunappa, R., Shekhar, S., Gurugnanam, B., Raju, P.L.N., and De, P. (2007) Road Network Analysis of Dehradun City Using High-Resolution Satellite Data and GIS. Journal of the Indian Society Of Remote Sensing, 35(3), 267-274.

34. Obafemi, A.A., Eludoyin, O.S., Opara, D.R. (2011) Road network assessment in Trans-Amadi, Port Harcourt in Nigeria using GIS. International Journal of Traffic and Transportation Engineering, 1(4), 257-264.

35. Sahitya, K.S., and Prasad, C.S.R.K. (2019) Modelling structural interdependent parameters of an urban road network using GIS. Spatial Information Research, DOI: 10.1007/s41324-019-00295-9.

36. Sahitya, K.S., and Prasad, C.S.R.K. (2020) Evaluation of opportunity based urban road network accessibility using GIS. Spatial Information Research, DOI: 10.1007/s41324-019-00309-6.

37. Sreeleka, M.G., Krishnamurthy, K., and Anjaneyulu, M.V.L.R. (2017) Fractal assessment of road transport system. European transport $\backslash$ Transport Europei, 65, paper number 5, 1-13.

38. Stojčić, M. (2018) Application of the ANFIS model in road traffic and transportation: a literature review from 1993 to 2018. Operational Research in Engineering Sciences: Theory and Applications, 1(1), 40-61, DOI: https://doi.org/10.31181/oresta19012010140s.

39. Sun, Z., Jia, P., Kato, H., and Hayashi, Y. (2007) Distributive Continuous Fractal analysis for urban transportation network. Journal of the Eastern Asia Society for transportation studies, 7.

40. Takagi, T., and Sugeno, M. (1985) Fuzzy identification of systems and its applications to modeling and control. IEEE Trans. on Systems, Man, and Cybernetics, 15:116-132.

41. Thipparat, T., and Thaseepetch, T. (2012) Application of Neuro-Fuzzy System to Evaluate Sustainability in Highway Design. International Journal of Modern Engineering Research (IJMER), 2(5), 4153-4158.

42. Transportation Research Board of the National Academies Circular Number E-C113, January (2007). Artificial Intelligence in Transportation, Information for application, ISSN 0097-8515.

43. Transportation Research Board of the National Academies Circular Number E-C168, November (2012). Artificial Intelligence applications to critical transportation issues, ISSN 097-8515.

44. Voort, M.D., Dougherty, M., and Watson, S. (1996) Combining Kohonen Maps with ARIMA Time Series Models to Forecast Traffic Flow. In: Transportation Research Part C, 4(5), 1996, 307-318.

45. Voženílek, V. (2009) Artificial intelligence and GIS: mutual meeting and passing. International Conference on Intelligent Networking and Collaborative Systems (INCOS 2009), 279-284. ISBN 978-1-4244-5165-4.

46. Wu, Y.H., and Miller, H.J. (2002) Computational tools for measuring space-time accessibility within transportation networks with dynamic flow. Journal of Transportation Statistics, 4(2/3), 1-14. 